\title{
Portography: a potentially fatal complication during endoscopic ultrasound-guided choledochoduodenostomy
}

Endoscopic ultrasound (EUS)-guided choledochoduodenostomy (EUS-CDS) is a safe alternative to transhepatic cholangiography when endoscopic retrograde cholangiopancreatography (ERCP) has failed or in patients with altered anatomy [1]. There are however several adverse events that can be life-threatening in some circumstances [1,2].

A 66-year-old woman presented with obstructive jaundice secondary to advanced pancreatic cancer. A gastrojejunal anastomosis had been previously performed to bypass a long duodenal stenosis. EUS-CDS was attempted because of difficulties in reaching the papilla. Temporary duodenal stenting as a bridge to ERCP was not considered given its high failure rate in the presence of tumor infiltration [3].

Under carbon dioxide insufflation, the dilated common bile duct (CBD) was punctured and bile was subsequently aspirated. The biliary tree was opacified and a 0.0025 -inch angled guidewire was inserted quite easily. A fistulotomy was performed with some temporary bleeding, which was controlled, at the puncture site. Shockingly, at the re-opacification check, the vascular portal system was recognized ( $\mathbf{F i g . 1}$ ), indicating that it had been catheterized, probably secondarily to erroneous guidewire manipulation. Given the absence of bleeding and the patient's stable vital parameters, the procedure was started again, and this time was successfully performed ( $\downarrow$ Video 1 ). The patient's recovery was uneventful and she left hospital 48 hours later.

Opacification and/or deep cannulation of the portal vein is a rare complication of ERCP [4], which occurs mainly in patients with biliopancreatic cancer during difficult cannulation or pre-cut, and may lead to potentially fatal air embolism. Balloon tamponade, covered stenting, or surgical repair is necessary in case of large defects. Being able to recognize an
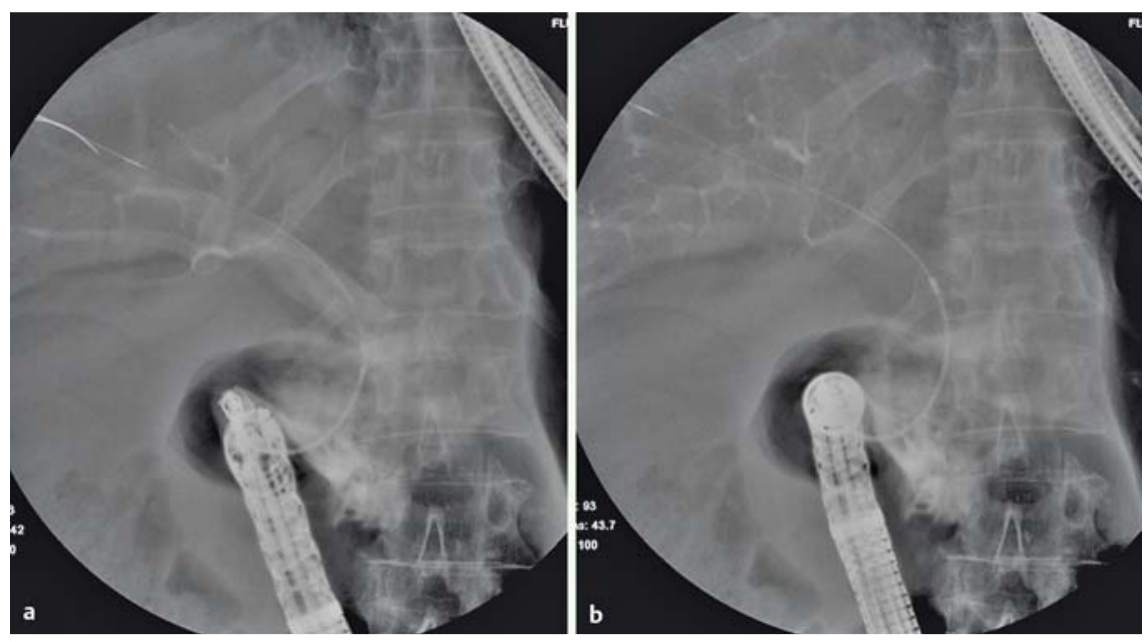

Fig. 1 Images of the re-opacification check during endoscopic ultrasound-guided choledochoduodenostomy (EUS-CDS) showing opacification of the vascular portal system with medium contrast wash-out, following accidental catheterization of the portal vein due, probably, to erroneous guidewire manipulation. erroneous catheterization during secondary opacification is mandatory and should result in the procedure being stopped immediately, not wrongly considering that it is the CBD.
This is the first report of portography as a complication during EUS-CDS. Certain technical steps are important to minimize the effects of this complication: gentle manipulation during deep insertion of the guidewire, followed by a

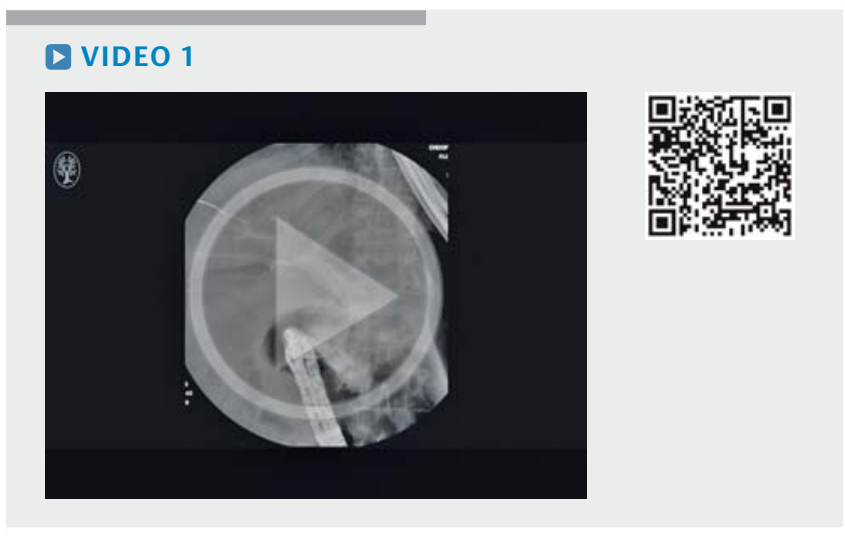

- Video 1: A 19-G needle was used to puncture the common bile duct, which was then opacified, followed by deep insertion of 0.0025 -inch angled guidewire and fistulotomy. The re-opacification check showed the procedure had been complicated by portography. The procedure was restarted and this time endoscopic ultrasound-guided choledochoduodenostomy (EUS-CDS) was successfully completed with delivery of a 6-cm fully covered biliary metal stent. 
check for biliary tree opacification, and carbon dioxide insufflation in order to minimize the risk of air embolism in case of vascular complications.

Endoscopy_UCTN_Code_CPL_1AK_2AC

Competing interests

None

The Authors

Gianfranco Donatelli, Jean-Loup Dumont, Stavros Dritsas, Thierry Tuszynski, Bertrand Marie Vergeau, Bruno Meduri Interventional Endoscopy Unit, Ramsay Générale de Santé, Hôpital Privé des Peupliers, Paris, France
Corresponding author

\section{Gianfranco Donatelli, MD}

Unité d'Endoscopie Interventionnelle, Ramsay Générale de Santé, Hôpital Privé des Peupliers, 8 Place de l'Abbé G. Hénocque, 75013, Paris, France,

Fax: +33-1-44165615,

donatelligianfranco@gmail.com

\section{References}

[1] Hara K, Yamao K, Mizuno N et al. Endoscopic ultrasonography-guided biliary drainage: Who, when, which, and how? World J Gastroenterol 2016; 21: 1297-1303

[2] Khashab MA, Messallam AA, Penas I et al. International multicenter comparative trial of transluminal EUS-guided biliary drainage via hepatogastrostomy vs. choledochoduo- denostomy approaches. Endosc Int Open 2016; 4: E175-E181

[3] Donatelli G, Cereatti F, Dumont J-L et al. Temporary duodenal stenting as a bridge to ERCP for inaccessible papilla due to duodenal obstruction: a retrospective study. Endosc Int Open 2016; 4: E957 - E963

[4] Kalaitzakis E, Stern N, Sturgess R. Portal vein cannulation: an uncommon complication of endoscopic retrograde cholangiopancreatography. World J Gastroenterol 2011; 17: $5131-5132$

\section{Bibliography}

DOI http://dx.doi.org/10.1055/s-0042-119396 Endoscopy 2017; 49: E16-E17

(c) Georg Thieme Verlag KG

Stuttgart · New York

ISSN 0013-726X 